ISSN: 2174-5609

DOI. https://doi.org/10.14198/INTURI2022.23.12

†T/ Investigaciones

$\triangle$ S TURISTICAS

Cita bibliográfica: Sustacha Melijosa, I., Baños Pino, J.F.y Del Valle Tuero, E. (2022). Análisis de la investigación sobre destinos turísticos inteligentes mediante la visualización de redes bibliométricas. Investigaciones Turísticas (23), pp. 266-289. https://doi.org/10.14198/INTURI2022.23.12

\title{
Análisis de la investigación sobre destinos turísticos inteligentes mediante la visualización de redes bibliométricas
}

An analysis of the research on smart tourism destinations based on bibliometric network visualisations

Inés Sustacha Melijosa (iD) Universidad de Oviedo, España

sustachaines@uniovi.es

José Francisco Baños Pino (iD) Universidad de Oviedo, España jbanos@uniovi.es

Eduardo Del Valle Tuero (iD, Universidad de Oviedo, España valleeduardo@uniovi.es

\section{RESUMEN}

El gran interés académico que ha despertado el paradigma smart ha hecho que la producción científica asociada a los destinos turísticos inteligentes se haya incrementado notablemente en los últimos años. El objetivo de este trabajo es estudiar su desarrollo y estructura mediante técnicas de análisis bibliométrico poniendo el foco en el destino, frente a investigaciones previas de corte más generalista. Este estudio se basa en 415 documentos indexados en Scopus hasta septiembre de 2020. En él, se analiza la evolución y la distribución geográfica del conocimiento sobre destinos inteligentes, además de las publicaciones, autores y documentos más influyentes. En concreto, se describe la estructura intelectual de esta literatura mediante una red de co-citación de autores construida con VOSviewer. Además, se ha realizado un análisis de co-ocurrencia de palabras clave para identificar los principales temas y tendencias, así como la existencia de brechas de conocimiento. Los mapas bibliométricos facilitan el análisis de la complejidad de la literatura sobre destinos inteligentes, ya que permiten visualizar las interrelaciones entre sus diversos elementos. Los resultados también apuntan los temas de interés más recientes y que merecen atención de cara a futuras investigaciones.

Palabras clave: Análisis bibliométrico; co-citación; co-ocurrencia de palabras; destinos turísticos inteligentes; visualización de red. 


\section{ABSTRACT}

The high level of academic interest in the smart paradigm has led to a significant increase in the scientific production associated with smart destinations in recent years. This paper aims to study its development and structure by using bibliometric analysis techniques and focusing on the destination, as opposed to previous research of a more generalist nature. This study is based on 415 documents indexed in Scopus until September 2020, analysing the evolution and geographical distribution of knowledge on smart destinations, as well as the most influential publications, authors and documents. More specifically, the intellectual structure of this literature has been described through an author co-citation network built with VOSviewer. In addition, a co-word analysis has been performed to identify the main topics and trends, and the existence of gaps in the knowledge. The bibliometric maps facilitate the analysis of the complexity of smart destination literature, as they allow us to visualise the interrelationships among its various elements. The results also indicate the most recent topics of interest which deserve attention in future research.

Keywords: Bibliometric analysis; co-citation; co-word analysis; smart tourism destinations; network visualisation.

\section{I.INTRODUCCIÓN}

El turismo es una importante fuente de ingresos para muchos destinos, que contribuye a crear empleo, promover el espíritu empresarial y desarrollar las economías locales. La industria del turismo es una de las más importantes a nivel mundial, representando el $7 \%$ de las exportaciones totales de bienes y servicios (WTO, 2021). En nuestro país tiene una importancia clave, que la actual crisis económica derivada de la COVID-19 ha puesto, si cabe, todavía más de manifiesto. En 2019 España ocupó el segundo puesto dentro de los destinos turísticos internacionales, tanto por el número de llegadas de turistas como por los ingresos generados (WTO, 2021). La contribución del turismo a la economía española alcanzó los 154.487 millones de euros, el $12,4 \%$ del PIB, mientras que las ramas características del turismo generaron 2,72 millones de puestos de trabajo, el 12,9\% del empleo total (Instituto Nacional de Estadística, 2020). A estas cifras hay que añadir su gran capacidad de arrastre y efecto multiplicador sobre otros sectores (Exceltur, 2018), así como su contribución para paliar el déficit comercial.

La generalización del uso de internet, la creciente utilización de dispositivos móviles, el desarrollo del e-commerce, las redes sociales, y, en definitiva, la irrupción de la nueva economía digital, han generado profundos cambios en los hábitos de los turistas y en su experiencia de viaje (Xiang et al., 2015), pero también nuevos modelos de negocio (Gretzel, Werthner, et al., 2015; Ivars-Baidal y Vera-Rebollo, 2019) y una mayor competencia entre los destinos (Neuhofer et al., 2014).

En este contexto, de uso intensivo de las tecnologías de la información en la producción y el consumo turísticos, por la necesidad de garantizar un desarrollo turístico equilibrado y competitivo, más sostenible, seguro y solidario, surge un nuevo concepto: el destino turístico inteligente. Aunque existen muchas definiciones (ver, por ejemplo, Boes et al., 2015, p. 394; Buhalis y Amaranggana, 2015, p. 378; Jovicic, 2016, p. 7; Lamsfus et al., 2015, p. 367), un destino turístico inteligente puede describirse como "un espacio turístico innovador, accesible para todos, consolidado sobre una infraestructura tecnológica de 
vanguardia que garantiza el desarrollo sostenible del territorio, facilita la interacción e integración del visitante con el entorno e incrementa la calidad de su experiencia en el destino y la calidad de vida de los residentes" (López de Ávila y García, 2013, p. 62; Segittur, 2015, p. 32). Por tanto, los destinos turísticos inteligentes se asientan en cuatro ejes fundamentales: innovación, tecnología, accesibilidad y sostenibilidad, a los que se añade un quinto: la gobernanza, al considerarse un factor esencial para el éxito de su implementación (Chourabi et al., 2012).

Los destinos turísticos inteligentes suponen un enfoque sistémico del destino, mucho más amplio que el derivado de la concepción tradicional. La revolución digital y las tecnologías de la comunicación han propiciado la aparición de este concepto en el que el conocimiento y la información son accesibles a todos los agentes, promoviendo la innovación continua (Jovicic, 2019).

Pese a su relativa juventud, la producción científica relacionada con los destinos turísticos inteligentes se ha incrementado notablemente, fruto del gran interés que ha despertado. Este hecho, unido a la disponibilidad y mejoras en la cobertura de las bases de datos bibliográficas como la Web of Science y Scopus, ha convertido a la bibliometría en una herramienta útil (Benckendorff, 2009) para abordar la estructura actual del conocimiento en esta área. Además, al tratarse de métodos basados en el análisis estadístico de datos cuantitativos proporcionados por la literatura científica, contribuyen a incrementar el rigor en las revisiones de la literatura minimizando el componente subjetivo del investigador (Zupic y Čater, 2015).

Por lo tanto, el propósito principal de este trabajo es investigar la producción científica asociada al concepto de destino turístico inteligente usando técnicas de análisis bibliométrico. Las investigaciones bibliométricas previas sobre turismo inteligente son de corte más generalista o en todo caso se centran en otros aspectos como el papel de las redes o los medios sociales. En cambio, la novedad de esta radica en que pone el foco en el destino, entendido como el espacio físico en el que se desarrolla la actividad turística y que tiene importantes implicaciones en cuanto a su gestión, para crear valor y mejorar la experiencia del turista (Neuhofer et al., 2012), incrementando la competitividad del destino (Koo et al., 2016). Además, la mayoría se limitan a unos pocos artículos publicados en revistas, mientras que el presente estudio comprende una base de conocimiento científico mucho más amplia considerando también capítulos de libros y actas de conferencias. Finalmente, este trabajo está especialmente enfocado en proporcionar una imagen gráfica o visualización de los resultados y su sistematización en grupos o clústeres de nodos similares y relacionados.

En cuanto a la estructura de este artículo, en primer lugar, se revisa el origen del concepto de los destinos inteligentes y la literatura existente sobre el turismo inteligente abordada mediante análisis bibliométricos. En segundo lugar, se detalla la metodología empleada en cuanto al diseño de la investigación, obtención de las referencias y el análisis, visualización e interpretación de resultados. A continuación, se exponen los resultados y finalmente, se discuten sus implicaciones y se exponen las conclusiones más relevantes. 


\section{II.REVISIÓN DE LA LITERATURA}

La idea de destino turístico inteligente está muy vinculada a la de smart city ${ }^{1}$, un renovado enfoque en la planificación y gestión urbana (Ivars-Baidal y Vera-Rebollo, 2019) que basa su estrategia en el uso de las tecnologías de la información y la comunicación (TIC) en áreas clave como la economía, el medio ambiente, la movilidad y la gobernanza para transformar las infraestructuras y los servicios de las ciudades (Bakici et al., 2013). En una smart city la tecnología está integrada en la ciudad y se combina con sus componentes sociales para mejorar la calidad de vida de los residentes y la eficiencia de los servicios urbanos (Buhalis y Amaranggana, 2013). Estos conceptos pueden trasladarse directamente a los destinos turísticos, que a menudo son zonas urbanas. Así, para Del Chiappa y Baggio (2015), el concepto de destino turístico inteligente resulta de la aplicación de los principios de las smart cities a los destinos turísticos, donde no sólo se considera a los residentes sino también a los turistas (Gretzel, Sigala, et al., 2015).

Los destinos turísticos inteligentes se sustentan en la implementación de herramientas tecnológicas para que tanto la demanda como la oferta puedan co-crear valor y enriquecer las experiencias de los turistas (Ballina et al., 2019; Brandão Cavalheiro et al., 2019) y, a la vez, generar beneficios y ventajas competitivas para las empresas y el destino (Boes et al., 2015). Internet y la computación en la nube han cambiado la forma de operar de las empresas simplificando el intercambio de datos, mejorando la toma de decisiones y su competitividad (Buhalis y Leung, 2018). Además, los productos que se consumen son cada vez más personalizados gracias a que la interacción de los turistas con el destino se hace a través de desarrollos tecnológicos portátiles y ubicuos (Shoval y Birenboim, 2019).

Este nuevo enfoque del destino ha despertado gran interés y la literatura científica sobre destinos turísticos inteligentes no ha parado de crecer por lo que resulta oportuno estudiar su desarrollo y estructura. Las técnicas de análisis bibliométrico son una buena herramienta para ello. La bibliometría es una parte de la cienciometría que aplica métodos matemáticos y estadísticos a la literatura científica y a los autores que la producen, con el objetivo de estudiar y analizar su actividad (Pritchard, 1969). En los últimos años ha habido un creciente interés en la aplicación de estos métodos en numerosas áreas de conocimiento. La investigación en turismo no ha sido ajena a este hecho (véase por ejemplo Benckendorff, 2009; Benckendorff y Zehrer, 2013; Hall, 2011; Koseoglu et al., 2016; Ruhanen et al., 2015) y el ámbito específico del turismo inteligente tampoco.

El artículo de Johnson y Samakovlis (2019) es uno de los primeros en proporcionar una mayor comprensión del turismo inteligente como tema de investigación al examinar su evolución en el contexto académico a través del análisis bibliométrico. Su principal hallazgo es que el turismo inteligente se configura y mejora a través de una red colaborativa de investigadores.

Vargas-Sánchez y Saltos (2019) caracterizan la investigación científica sobre turismo inteligente y su interrelación con las redes sociales para comprender mejor su papel.

\footnotetext{
${ }^{1}$ Para una definición de smart city véase Giffinger et al. (2007) y Caragliu et al. (2011). En Albino et al. (2015) se hace una revisión bibliográfica en profundidad, identificando las principales dimensiones y elementos que caracterizan una ciudad inteligente.
} 
Realizaron una búsqueda sistemática en Web of Science y Scopus, considerando un total de 61 documentos, la mayoría artículos de revistas. Presentan una imagen detallada de la investigación en la intersección mencionada: evolución en el tiempo; autores, instituciones y países más prolíficos; revistas, conferencias y editoriales que han prestado más atención a este tema; tipo de investigación realizada, objetos de estudio, metodologías y principales contribuciones.

También Nusair (2020) pone el foco en la importancia indiscutible del papel de los medios de comunicación sociales, abordando su rápido crecimiento en la investigación en turismo. Analizó 601 documentos publicados entre 2002 y 2018 en revistas de hostelería y turismo. El período 2007-2012 marca el inicio de las redes sociales como un nuevo fenómeno en este ámbito, mientras que entre 2013 y 2018 emergen nuevas tendencias como big data, e-turismo, green experience y el turismo inteligente. Este artículo también propone un ciclo de vida de los temas clave relacionados con la investigación en redes sociales.

Mehraliyev et al. (2020) utilizan métodos mixtos que combinan técnicas cualitativas y cuantitativas para identificar las principales tendencias y articular dominios del conocimiento en la investigación del turismo inteligente. Este estudio analizó 86 artículos, identificando 11 temas en la investigación sobre turismo inteligente y 4 dominios del conocimiento, entre ellos los enfocados a los destinos inteligentes y al papel de las tecnologías.

En uno de los trabajos más recientes de análisis bibliométrico, Bastidas-Manzano et al. (2020), revisan el desarrollo de los conceptos de smart cities y destinos turísticos inteligentes. Analizaron 258 documentos publicados entre 2013 y 2019 en la Web of Science y otras fuentes estrechamente relacionadas con las tecnologías de la información en la hostelería y el turismo como el Journal of Information Technology \& Tourism (JITT), el Journal of Hospitality and Tourism Technology (JHTT) y las actas de la conferencia ENTER. Mediante un diagrama estratégico realizado con el software SciMAT (Cobo et al., 2012), identifican los principales temas que merecen ser considerados por los profesionales y los académicos en la investigación futura sobre destinos inteligentes.

Finalmente, aunque en sentido estricto no usan técnicas de análisis bibliométrico, merece la pena mencionar el trabajo de Celdrán-Bernabeu et al. (2018) que contabilizan y categorizan la producción científica relacionada con el turismo inteligente aplicando un estudio de mapeo sistemático, novedoso en el ámbito del turismo.

En la Tabla 1 se sintetizan todas estas aportaciones a través de sus objetivos, los datos y la metodología que han empleado, así como los principales resultados que han obtenido. 
Tabla 1: Aproximaciones bibliométricas a la investigación sobre turismo inteligente

\begin{tabular}{|c|c|c|c|c|}
\hline Autor/es (año) & Objetivo & Datos & Metodología & Resultados \\
\hline $\begin{array}{l}\text { Celdrán-Bernabeu } \\
\text { et al. (2018) }\end{array}$ & $\begin{array}{l}\text { Identificar y caracterizar la } \\
\text { producción científica asociada al } \\
\text { concepto de turismo inteligente. }\end{array}$ & $\begin{array}{l}\text { Scopus. } \\
162 \text { artículos, capítulos de libros y } \\
\text { conferencias. } \\
\text { Idioma: inglés. } \\
\text { Período: } 2000-2017 .\end{array}$ & Estudio de mapeo sistemático & $\begin{array}{l}\text { Caracterización de las publicaciones } \\
\text { que ayuda a interpretar brechas en el } \\
\text { conocimiento y oportunidades para } \\
\text { publicaciones futuras. }\end{array}$ \\
\hline $\begin{array}{l}\text { Johnson y } \\
\text { Samakovlis (2019) }\end{array}$ & $\begin{array}{l}\text { Examinar la producción del } \\
\text { conocimiento sobre turismo } \\
\text { inteligente, revelando su desarrollo a } \\
\text { través de redes de conocimiento. }\end{array}$ & $\begin{array}{l}\text { Web of Science Core Collection y Scopus. } \\
247 \text { artículos. } \\
\text { Idioma: inglés. } \\
\text { Período: } 2000-2018 .\end{array}$ & $\begin{array}{l}\text { Co-autoría } \\
\text { Co-citación (autores) } \\
\text { Co-ocurrencia de palabras } \\
\text { clave }\end{array}$ & $\begin{array}{l}\text { El turismo inteligente se configura y } \\
\text { mejora a través de una red } \\
\text { colaborativa de investigadores. }\end{array}$ \\
\hline $\begin{array}{l}\text { Vargas-Sánchez y } \\
\text { Saltos (2019) }\end{array}$ & $\begin{array}{l}\text { Revisar la producción científica sobre } \\
\text { turismo inteligente y su interrelación } \\
\text { con las redes sociales. }\end{array}$ & $\begin{array}{l}\text { Web of Science, Scopus, Abi/Inform } \\
\text { Collection, Academia.edu, Google Scholar y } \\
\text { Dialnet. } \\
61 \text { artículos, capítulos de libros, conferencias } \\
\text { y tesis. } \\
\text { Idioma: inglés y español. } \\
\text { Hasta diciembre de } 2018 \text {. }\end{array}$ & $\begin{array}{l}\text { Número de documentos } \\
\text { Citación (documentos) }\end{array}$ & $\begin{array}{l}\text { Caracterización de la investigación } \\
\text { sobre turismo inteligente y su } \\
\text { interrelación con las redes sociales. }\end{array}$ \\
\hline $\begin{array}{l}\text { Bastidas-Manzano } \\
\text { et al. (2020) }\end{array}$ & $\begin{array}{l}\text { Ofrecer una visión general de la } \\
\text { evolución de los destinos turísticos } \\
\text { inteligentes, su alcance actual y sus } \\
\text { potenciales ámbitos de interés. }\end{array}$ & $\begin{array}{l}\text { Web of Science y otras publicaciones sobre } \\
\text { tecnologías de la información relacionadas con } \\
\text { la hostelería y el turismo. } \\
258 \text { artículos, artículos de revisión, libros, } \\
\text { capítulos de libros y conferencias } \\
\text { Idioma: inglés. } \\
\text { Período: } 2013-2019 .\end{array}$ & $\begin{array}{l}\text { Número de documentos } \\
\text { Co-ocurrencia de palabras } \\
\text { clave }\end{array}$ & $\begin{array}{l}\text { Identificación de los principales temas } \\
\text { que merecen ser considerados por los } \\
\text { profesionales y los académicos en la } \\
\text { investigación futura sobre destinos } \\
\text { inteligentes. }\end{array}$ \\
\hline $\begin{array}{l}\text { Mehraliyev et al. } \\
\text { (2020) }\end{array}$ & $\begin{array}{l}\text { Identificar los temas y dominios del } \\
\text { conocimiento más destacados en la } \\
\text { investigación del turismo inteligente. }\end{array}$ & $\begin{array}{l}\text { Web of Science, Scopus, y EBSCOhost } \\
86 \text { artículos. } \\
\text { Idioma: inglés. } \\
\text { Período: hasta } 2018 .\end{array}$ & $\begin{array}{l}\text { Método mixto que combina } \\
\text { técnicas de revisión } \\
\text { cualitativa y co-citación } \\
\text { (documentos) }\end{array}$ & $\begin{array}{l}\text { Identificaron } 11 \text { temas y } 4 \text { dominios del } \\
\text { conocimiento y las principales lagunas } \\
\text { en la investigación del turismo } \\
\text { inteligente. }\end{array}$ \\
\hline Nusair (2020) & $\begin{array}{l}\text { Proporcionar un análisis } \\
\text { bibliométrico exhaustivo del estado } \\
\text { actual de la literatura sobre los } \\
\text { medios sociales. }\end{array}$ & $\begin{array}{l}\text { EBSCOhost y Science Direct } \\
601 \text { artículos. } \\
\text { Período: 2002-2018. }\end{array}$ & $\begin{array}{l}\text { Número de documentos } \\
\text { Co-ocurrencia de palabras } \\
\text { clave }\end{array}$ & $\begin{array}{l}\text { Resumen de los resultados en un } \\
\text { ciclo de vida de las tendencias } \\
\text { temáticas de los medios sociales. }\end{array}$ \\
\hline
\end{tabular}

Elaboración propia 


\section{III.METODOLOGÍA}

Zupic y Čater (2015) plantean un flujo de trabajo para realizar estudios de mapeo científico con métodos bibliométricos basado en cinco pasos: diseño de la investigación, obtención de las referencias, análisis, visualización e interpretación de los resultados. El uso de software específico hace que las tres últimas etapas puedan condensarse en una única fase.

\subsection{Diseño de la investigación}

La etapa inicial, de diseño de la investigación, pasa por definir cuáles son las preguntas relevantes de la investigación y elegir los métodos bibliométricos más adecuados que permitan dar respuesta a esas preguntas. La Tabla 2 muestra ambos aspectos.

Tabla 2: Diseño de la investigación

\begin{tabular}{|l|c|}
\hline \multicolumn{1}{|c|}{ Preguntas de la investigación } & Métodos bibliométricos \\
\hline $\begin{array}{l}\text { PI1: ¿Cuál es la evolución del conocimiento sobre } \\
\text { destinos turísticos inteligentes? }\end{array}$ & Número de documentos/Citación \\
\hline $\begin{array}{l}\text { PI2: ¿Cuál es la distribución geográfica del conocimiento } \\
\text { sobre destinos turísticos inteligentes? }\end{array}$ & Número de documentos/Citación \\
\hline $\begin{array}{l}\text { PI3: ¿Qué publicaciones, autores y documentos han } \\
\text { influido en la investigación sobre destinos turísticos } \\
\text { inteligentes? }\end{array}$ & $\begin{array}{c}\text { Número de documentos (publicaciones } \\
\text { y autores)/Citación (publicaciones, } \\
\text { autores y documentos) }\end{array}$ \\
\hline $\begin{array}{l}\text { PI4: ¿Cuál es la estructura intelectual de la literatura } \\
\text { sobre destinos turísticos inteligentes? }\end{array}$ & \multicolumn{1}{c|}{ Co-citación (autores) } \\
\hline $\begin{array}{l}\text { PI5: ¿Cuál es la estructura conceptual y temática de la } \\
\text { literatura sobre destinos turísticos inteligentes? }\end{array}$ & Co-ocurrencia de palabras \\
\hline
\end{tabular}

Elaboración propia

La citación es fundamentalmente una medida del impacto, por lo que su principal interés reside en identificar las publicaciones, autores y documentos más influyentes en un campo de investigación concreto (Zupic y Čater, 2015).

La co-citación es una relación de co-ocurrencia que se da cuando dos ítems de la literatura existente son citados juntos por un tercero (Miguel et al., 2007), en el caso que nos ocupa, un autor que cita a otros dos (White y Griffith, 1981). Este tipo de análisis asume que entre dos o más autores que son co-citados, existe una afinidad temática y que cuanto mayor sea la frecuencia de co-citación, mayor será la similitud entre ellos. El objetivo es determinar quiénes son los investigadores centrales de una determinada disciplina, en función de las cocitas de los demás.

El análisis de co-ocurrencia de palabras está basado en la aparición conjunta de dos términos en un texto con el propósito de determinar la estructura conceptual y temática de un dominio científico (Callon et al., 1983). Cuanto mayor sea la frecuencia de aparición conjunta de las palabras, mayor será su vinculación (Miguel et al., 2008). Como resultado se obtiene una red de temas y sus interrelaciones, que representan la estructura conceptual de un campo.

\subsection{Obtención de las referencias bibliométricas}

Para obtener las referencias se ha elegido como fuente Scopus, debido a su mayor cobertura en revistas científicas relacionadas con el turismo (Hall, 2011; McKercher, 2007). 
Scopus, editada por Elsevier, es la mayor base de datos de citas y resúmenes de bibliografía revisada por pares con más de 73 millones de registros publicados en 24.000 revistas científicas, procedentes de más de 5.000 editoriales. Se realizó una consulta el 15 de septiembre de 2020, con el término Smart* Touris* Destination* (para incluir variaciones de términos con la misma raíz) en el campo "TITLE-ABS-KEY" (busca en el título, el resumen, y las palabras clave), devolviendo 467 documentos como resultado.

Los resultados se han sometido a un proceso de filtrado ${ }^{2}$ en función de varios aspectos como el idioma, el periodo de tiempo y el tipo de documento (Aguinis et al., 2018; Zupic y Čater, 2015). Se han incluido sólo documentos en inglés dado que los algoritmos de procesamiento de lenguaje natural (PLN) que utiliza el software empleado para el análisis no admiten otros idiomas. No se ha fijado ningún límite temporal, asumiendo el posible sesgo que supone no tener el año 2020 completo debido, por un lado, a la fecha de la consulta y por otro, al desfase temporal entre la publicación de un trabajo y su indización en Scopus. Finalmente, aunque los artículos publicados en revistas científicas son los únicos considerados como "conocimiento certificado" al ser sometidos a la revisión crítica y a la aprobación de otros investigadores (Ramos-Rodríguez y Ruiz-Navarro, 2004), se ha optado por incluir además los capítulos de libros y actas de conferencias dado que nuestro objetivo es analizar la producción científica sobre destinos turísticos inteligentes en un sentido amplio. En total, se ha obtenido un resultado final de 415 referencias, de las cuales, la mayoría, 279 son artículos, 117 son trabajos presentados en congresos y 19 capítulos de libro.

\subsection{Análisis, visualización e interpretación de los resultados}

Las referencias que hacen unos autores a otros, así como entre revistas o cualquier otro tipo de documentos pueden representarse mediante un grafo con estructura de red (Spinak, 1996) que consta de dos elementos fundamentales: los nodos, que representan los documentos, autores, palabras clave, etc. y los enlaces, llamados también aristas, que conectan entre sí uno o más nodos. Con el fin de evitar duplicidades en los registros y corregir inconsistencias, ha sido necesario elaborar un tesauro para autores y palabras clave.

Para construir, visualizar y explorar redes bibliométricas se ha utilizado el software libre VOSviewer, desarrollado en el Centre for Science and Technology Studies de la Universidad de Leiden (Van Eck y Waltman, 2010, 2014). Este programa proporciona visualizaciones de redes bibliométricas mediante mapas basados en la distancia, de modo que la distancia entre los nodos refleja la fuerza de la relación entre los mismos. Se ha optado por el método de recuento fraccional ${ }^{3}$, ya que ha demostrado ser más útil que el completo (Perianes-Rodríguez et al., 2016). El programa efectúa un análisis clúster, agrupando cada uno de los nodos en función de patrones de proximidad o distancia, obteniendo como resultado

\footnotetext{
${ }^{2}$ La expresión final que contiene todos los filtros utilizados para obtener las referencias en Scopus ha sido: TITLEABS-KEY (smart* AND touris* AND destination*) AND (LIMIT-TO (LANGUAGE, "English") AND (LIMIT-TO (DOCTYPE, “ar") OR LIMIT-TO (DOCTYPE, “cp") OR LIMIT-TO (DOCTYPE, “ch").

${ }^{3}$ En el recuento completo (full counting) cada co-autoría, co-ocurrencia, acoplamiento bibliográfico o enlace de co-citación tiene el mismo peso. En el recuento fraccional (fractional counting) el peso de cada enlace está fraccionado, por ejemplo, si un autor es co-autor de un documento con otros 10, cada uno de los 10 enlaces de co-autoría tiene un peso de $1 / 10$. No obstante, en los análisis efectuados en este trabajo no se han encontrado grandes diferencias aplicando los dos tipos de recuentos.
} 
grupos o clústeres de nodos similares, diferenciados por colores. El proceso concluye con la descripción e interpretación de los resultados.

\section{IV.RESULTADOS}

Para dar respuesta a la primera pregunta, relativa a la evolución del conocimiento sobre destinos inteligentes, se utilizarán indicadores de producción basados en el recuento de publicaciones y de citas. Los 415 documentos analizados en este trabajo han dado lugar a un total de 3.945 citas. A partir de 2015, año en el que aparecen las definiciones más aceptadas de destinos turísticos inteligentes se produce un despegue de la literatura, alcanzándose la cifra máxima en 2019 con un total de 112 documentos. Como ya se ha indicado, el año 2020 no está completo, pero transcurridos ocho meses y medio ya hay indexados 72 trabajos (Gráfico 1)

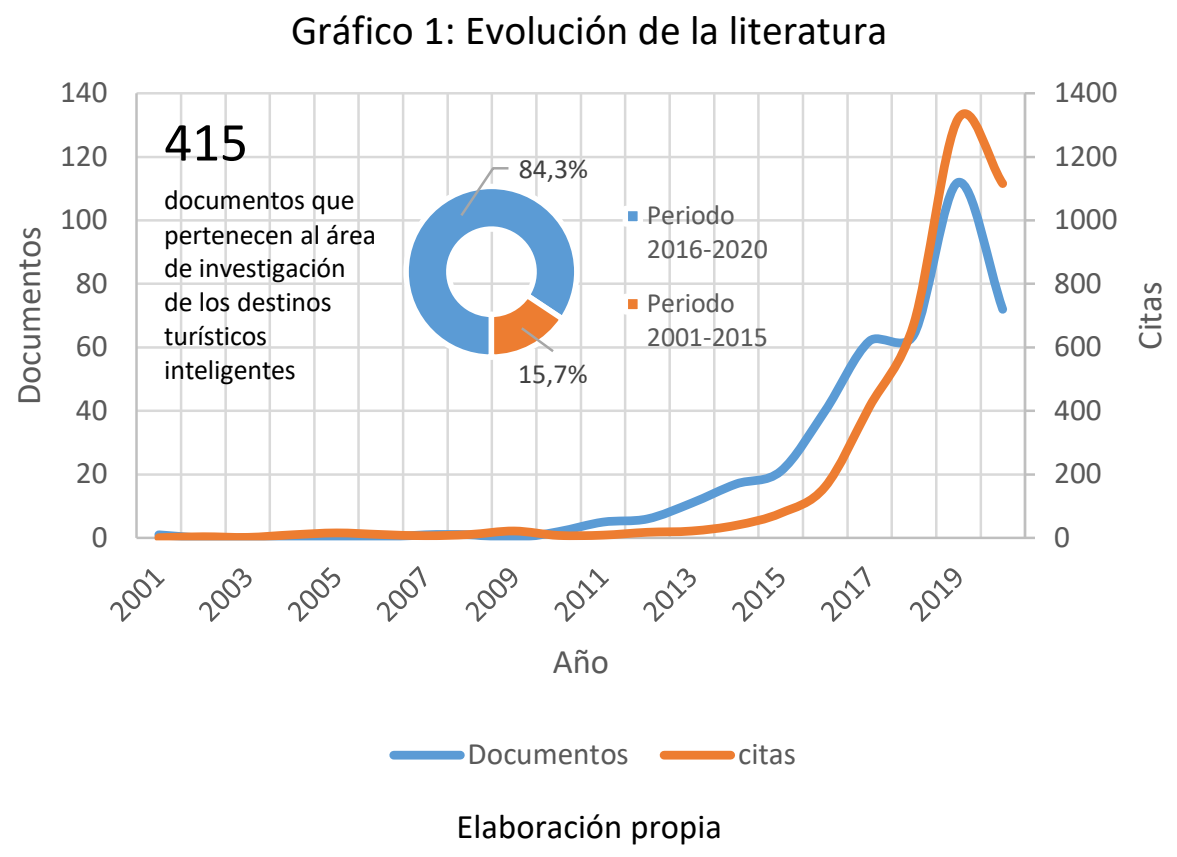

El

Gráfico 2 muestra la distribución del número de documentos y de citas por países y da una idea de cómo se distribuye geográficamente el conocimiento sobre destinos inteligentes, proporcionando respuesta a la segunda de las preguntas de investigación. Los trabajos provienen de 67 países diferentes, aunque parece que existe un mayor interés en Europa y Asia, que concentran respectivamente un $43,9 \%$ y un $38 \%$ de la producción científica mundial. España es el país más prolífico en cuanto a número de documentos, seguido por Estados Unidos e Italia. Sin embargo, por número de citas, Estados Unidos es el país más influyente, situándose a continuación Corea del Sur y el Reino Unido. 
Gráfico 2: Distribución geográfica del conocimiento

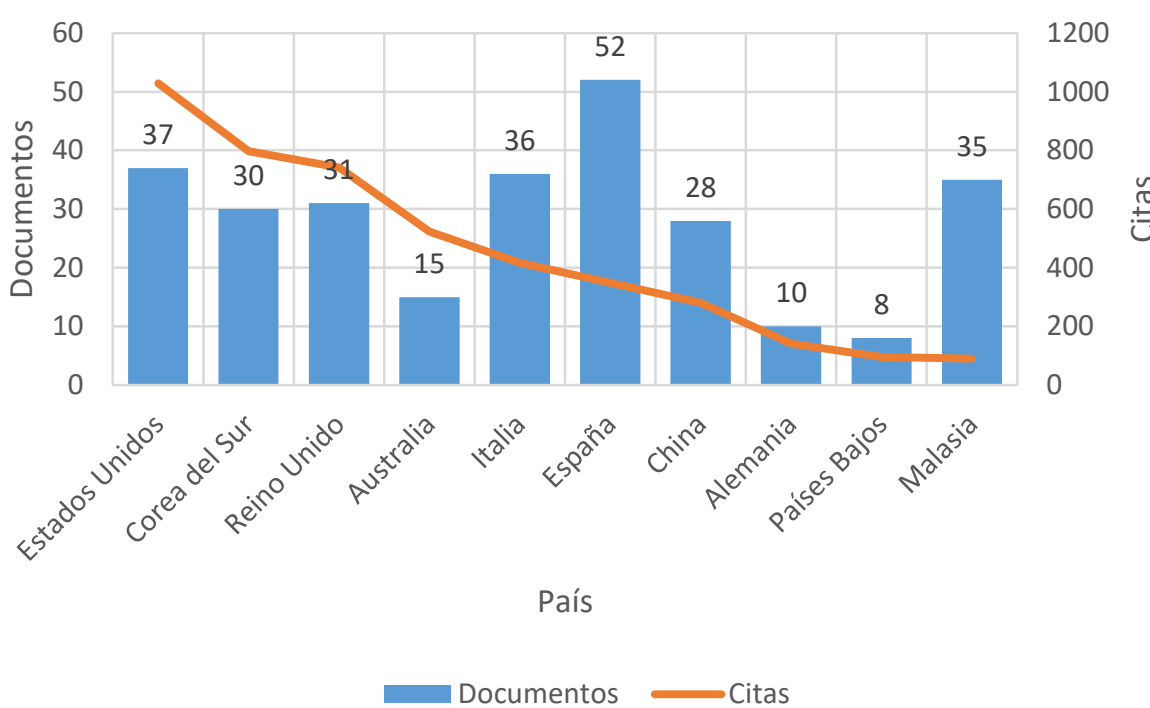

Elaboración propia

La tercera pregunta de investigación se refiere a cuáles son las publicaciones, los autores y los documentos que han tenido mayor influencia en la investigación sobre destinos inteligentes. Entre las 227 publicaciones diferentes identificadas destacan tres revistas científicas de primer nivel: Journal of Destination Marketing and Management, con un total de 521 citas, seguida de Electronic Markets y de Tourism Management, con 374 y 216 citas respectivamente. No obstante, por número de artículos sobresalen Sustainability e International Journal of Tourism Cities. Es preciso indicar que hay revistas donde se han editado muy pocos trabajos pero que han tenido un gran impacto como Electronic Markets, en la que se publicó el influyente artículo de Gretzel, Sigala, et al. (2015) y que sienta las bases del turismo inteligente (Tabla 3). Además, se aprecia una cierta concentración en torno a determinadas revistas y publicaciones, ya que solo 17 han publicado más de 5 trabajos relacionados con los destinos turísticos inteligentes.

Tabla 3: Principales publicaciones

\begin{tabular}{|c|l|c|c|c|}
\hline Ranking & \multicolumn{1}{|c|}{ Fuente } & Documentos & Citas & $\begin{array}{c}\text { Cuartil } \\
\text { Scopus }\end{array}$ \\
\hline 1 & Journal of Destination Marketing and Management & 11 & 521 & $\mathrm{Q} 1$ \\
\hline 2 & Electronic Markets & 1 & 374 & $\mathrm{Q} 1$ \\
\hline 3 & Tourism Management & 6 & 216 & $\mathrm{Q} 1$ \\
\hline 4 & Current Issues in Tourism & 5 & 208 & $\mathrm{Q} 1$ \\
\hline 5 & Sustainability & 31 & 201 & $\mathrm{Q} 2$ \\
\hline
\end{tabular}




\begin{tabular}{|c|l|c|c|c|}
\hline 6 & International Journal of Tourism Cities & 13 & 158 & Q3 \\
\hline 7 & IEE Conference Publication & 1 & 151 & - \\
\hline 8 & Technological Forecasting and Social Change & 5 & 148 & Q1 \\
\hline 9 & Journal of Travel Research & 8 & 137 & Q1 \\
\hline 10 & Journal of the American Planning Association & 1 & 134 & Q1 \\
\hline
\end{tabular}

Elaboración propia

Analizando el número de veces que han sido citados los autores es posible determinar quiénes son los expertos en un campo de investigación específico. En nuestro caso, una vez eliminadas las duplicidades, se han identificado un total de 1.112 autores diferentes entre los que destacan Chulmo Koo, de la Kyung Hee University de Corea del Sur y Ulrike Gretzel, de la University of Southern California en Estados Unidos. Sin embargo, el más prolífico es Nahmo Chung, también de la Kyung Hee University de Corea del Sur. Se observa la existencia de autores que han publicado muy pocos trabajos pero que han tenido un gran impacto como Marianna Sigala, de la University of South Australia y Zheng Xiang, de Virginia Tech (Tabla 4).

Tabla 4: Principales autores

\begin{tabular}{|c|l|c|c|}
\hline Ranking & \multicolumn{1}{|c|}{ Autor } & Documentos & Citas \\
\hline 1 & Koo, C. & 8 & 537 \\
\hline 2 & Gretzel, U. & 8 & 482 \\
\hline 3 & Sigala, M. & 1 & 374 \\
\hline 4 & Xiang, Z. & 1 & 374 \\
\hline 5 & Buhalis, D. & 6 & 333 \\
\hline 6 & Chung, N. & 13 & 304 \\
\hline 7 & Li, Y. & 2 & 205 \\
\hline 8 & Cherrett, T. & 2 & 171 \\
\hline 9 & Davies, N. & 2 & 171 \\
\hline 10 & Dickinson, J.E. & 2 & 171 \\
\hline
\end{tabular}

Elaboración propia

Mientras 983 autores han publicado solamente un documento, tan solo 10 firman más de 5 y únicamente 4 (Nahmo Chung, May-Chiun Lo, Ulrike Gretzel y Chulmo Koo), más de 8. En este sentido, puede decirse que se cumple la ley de productividad científica de Lotka (1926), pues la mayoría de los autores publican muy pocos documentos, mientras que un número reducido firman la mayoría de los trabajos relevantes, contribuyendo en mayor medida al desarrollo de este campo.

A través del análisis de citación directa también es posible determinar cuál es el núcleo de documentos principales en una disciplina, es decir, cuál es la "lista de lectura" recomendada (Zupic y Čater, 2015). En el caso que nos atañe destaca claramente el artículo de Gretzel, Sigala, et al. (2015) donde se define el turismo inteligente y los destinos inteligentes como un componente del mismo. A continuación, se sitúa el trabajo de Dickinson et al. (2014) sobre el importante papel de las apps para smartphones en el ámbito de los viajes y el de Poslad et al. (2001) sobre creación de servicios móviles para el turismo fáciles de usar y personalizados. Cabe indicar que la distribución de citas es sesgada, con un pequeño número de artículos altamente citados y muchos artículos con relativamente pocas o ninguna cita. Así, los 10 documentos más citados concentran 1.510 citas, el 38,3\%, mientras que 199 documentos, el $48 \%$, reciben una o ninguna. No obstante, el mero recuento de citas no aporta 
toda la información. El impacto de citas normalizado por campo o Field-Weighted Citation Impact ( $\mathrm{FWCl}$ ) pone las citas en contexto proporcionando el impacto de la citación de un documento en relación con el promedio de documentos similares, por categoría científica, año de publicación, y tipo de documento. Un valor superior a 1 significa que el documento es más citado de lo esperado. Los valores más elevados de esta métrica los obtienen Gretzel, Sigala, et al. (2015), McDonald (2015) y Dickinson et al. (2014) (Tabla 5).

Tabla 5. Principales documentos

\begin{tabular}{|c|c|c|c|c|c|}
\hline Ranking & Autor & Título & Fuente & Citas & $\mathrm{FWCl}$ \\
\hline 1 & $\begin{array}{l}\text { Gretzel, Sigala, } \\
\text { et al. (2015) }\end{array}$ & $\begin{array}{l}\text { Smart tourism: foundations and } \\
\text { developments }\end{array}$ & Electronic Markets & 374 & 23,50 \\
\hline 2 & $\begin{array}{l}\text { Dickinson } \\
\text { et al. (2014) }\end{array}$ & $\begin{array}{l}\text { Tourism and the smartphone app: } \\
\text { capabilities, emerging practice and } \\
\text { scope in the travel domain }\end{array}$ & $\begin{array}{l}\text { Current Issues in } \\
\text { Tourism }\end{array}$ & 152 & 10,62 \\
\hline 3 & $\begin{array}{l}\text { Poslad et al. } \\
(2001)\end{array}$ & $\begin{array}{l}\text { CRUMPET: Creation of user-friendly } \\
\text { mobile services personalised for } \\
\text { tourism }\end{array}$ & $\begin{array}{l}\text { IEE Conference } \\
\text { Publication }\end{array}$ & 151 & 8,05 \\
\hline 4 & $\begin{array}{l}\text { McDonald } \\
(2015)\end{array}$ & $\begin{array}{l}\text { Are Millennials Really the "Go- } \\
\text { Nowhere" Generation? }\end{array}$ & $\begin{array}{l}\text { Journal of the } \\
\text { American Planning } \\
\text { Association }\end{array}$ & 134 & 11,82 \\
\hline 5 & $\begin{array}{l}\text { Chung et al. } \\
(2015)\end{array}$ & $\begin{array}{l}\text { Tourists' intention to visit a } \\
\text { destination : The role of augmented } \\
\text { reality (AR) application for a } \\
\text { heritage site }\end{array}$ & $\begin{array}{l}\text { Computers in Human } \\
\text { Behavior }\end{array}$ & 127 & 6,24 \\
\hline 6 & $\begin{array}{l}\text { Buhalis y } \\
\text { Foerste (2015) }\end{array}$ & $\begin{array}{l}\text { SoCoMo marketing for travel and } \\
\text { tourism: Empowering co-creation } \\
\text { of value }\end{array}$ & $\begin{array}{l}\text { Journal of Destination } \\
\text { Marketing and } \\
\text { Management }\end{array}$ & 126 & 5,74 \\
\hline 7 & $\begin{array}{l}\text { Mariné-Roig y } \\
\text { Antón Clavé } \\
\text { (2015) }\end{array}$ & $\begin{array}{l}\text { Tourism analytics with massive user- } \\
\text { generated content: A case study of } \\
\text { Barcelona. }\end{array}$ & $\begin{array}{l}\text { Journal of Destination } \\
\text { Marketing and } \\
\text { Management }\end{array}$ & 119 & 8,60 \\
\hline 8 & $\begin{array}{l}\text { Boes et al. } \\
(2016)\end{array}$ & $\begin{array}{l}\text { Smart tourism destinations: } \\
\text { ecosystems for tourism destination } \\
\text { competitiveness. }\end{array}$ & $\begin{array}{l}\text { International Journal } \\
\text { of Tourism Cities }\end{array}$ & 116 & 9,18 \\
\hline 9 & $\begin{array}{l}\text { Wang et al. } \\
(2013)\end{array}$ & $\begin{array}{l}\text { China's "smart tourism destination» } \\
\text { initiative: A taste of the service- } \\
\text { dominant logic }\end{array}$ & $\begin{array}{l}\text { Journal of Destination } \\
\text { Marketing and } \\
\text { Management }\end{array}$ & 110 & 3,67 \\
\hline 10 & $\begin{array}{l}\text { Del Chiappa y } \\
\text { Baggio (2015) }\end{array}$ & $\begin{array}{l}\text { Knowledge transfer in smart } \\
\text { tourism destinations: Analyzing the } \\
\text { effects of a network structure }\end{array}$ & $\begin{array}{l}\text { Journal of Destination } \\
\text { Marketing and } \\
\text { Management }\end{array}$ & 101 & 6,17 \\
\hline
\end{tabular}
Elaboración propia

Para responder a la cuarta pregunta de investigación, referente a la estructura intelectual de la literatura sobre destinos turísticos inteligentes, se realizó un análisis de co-citación de autores, una relación de co-ocurrencia que se da cuando dos autores son citados a la vez por un tercero. Por tanto, el análisis se aleja ahora de los documentos de partida para examinar las características de las citaciones proporcionadas en la bibliografía de cada documento. El software VOSviewer identificó un total de 20.304 autores en la bibliografía de los 415 documentos de la muestra. Fijando un umbral de al menos 25 citas compartidas para mantener la red relativamente despejada y facilitar su interpretación, se obtiene un mapa con 130 autores (Figura1). 
Figura 1: Mapa de co-citación de autores

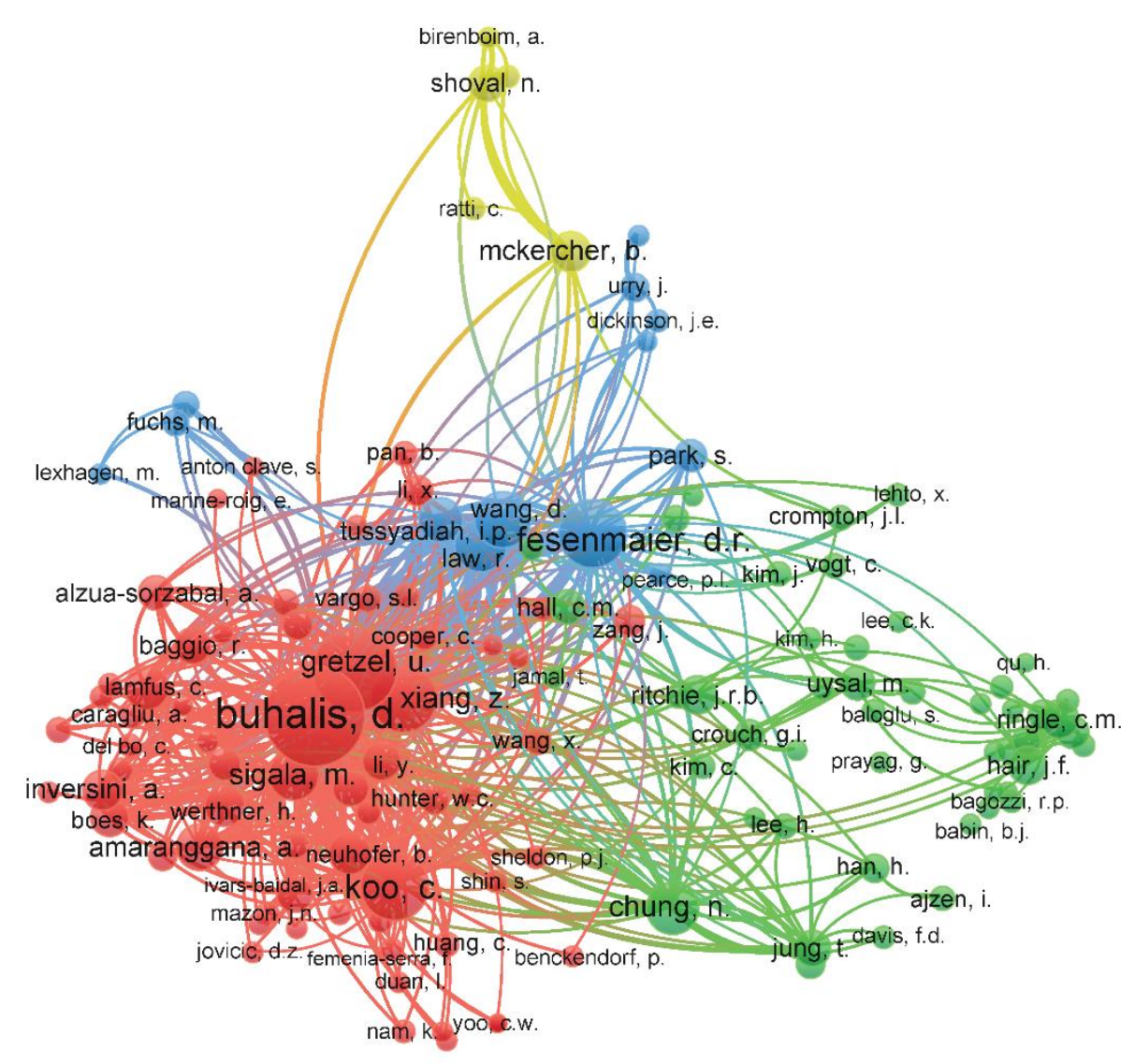

Elaboración propia

Cada nodo representa un autor y su tamaño indica el número de citas compartidas. Los enlaces entre nodos representan la cantidad de veces que un autor es citado por otro. Además, al ser un mapa basado en la distancia, cuanto menor sea la distancia entre dos nodos, mayor será su relación. Dimitrios Buhalis (673), Ulrike Gretzel (469), Chulmo Koo (316), Daniel R. Fesenmaier (313), Zheng Xiang (289) y Dan Wang (214) son los autores que más frecuentemente son co-citados.

En el mapa, los autores se agrupan en 4 clústeres identificados por diferentes colores. El clúster 1 (rojo), en la parte izquierda de la red, está formado por 63 autores, conocidos por su trabajo en tecnologías de la información aplicadas al turismo. Dimitrios Buhalis juega un papel central y los vínculos entre su trabajo y el de Ulrike Gretzel, Chulmo Koo y Zheng Xiang son notables. El clúster 2 (verde) es un grupo bastante disperso que incluye 48 autores entre los que destaca Nahmo Chung, muy próximo al clúster 1 y que hace de puente entre los dos grupos. En él, hay autores claramente orientados hacia la gestión y la planificación estratégica como Colin Michael Hall. También se encuentran J. R. Brent Ritchie, y mucho más alejados, John Crompton y el subgrupo en torno a Christian M. Ringle y Joe F. Hair, focalizados en la modelización de ecuaciones estructurales. El clúster 3 (azul) contiene 14 autores entre los que sobresale Daniel R. Fesenmaier, que tiene importantes vínculos con Dan Wang, Rob Law e lis Tussyadiah. El trabajo de estos autores está orientado principalmente hacia el destination marketing y la influencia de las tecnologías de la información y la comunicación en el 
comportamiento del consumidor y la experiencia de viaje, por eso se sitúan relativamente próximos a los autores más relevantes del clúster 1 (rojo). En este grupo también se encuentra el sociólogo John Urry, aunque ocupando una posición menos central. El clúster 4 (amarillo) agrupa a 5 autores y tiene un carácter mucho más periférico y disperso. Bob McKercher sirve de puente a autores aún más periféricos como Noam Shoval o Amit Birenboim con una perspectiva enfocada hacia la geografía y el territorio.

Para dar respuesta a la quinta pregunta de investigación (cuál es la estructura conceptual y temática de la literatura sobre destinos turísticos inteligentes) se ha realizado un análisis de co-ocurrencia de palabras para definir cuáles son los temas de mayor interés para los académicos. Mientras que en el análisis de co-citación las relaciones se infieren indirectamente a partir de las referencias bibliográficas de cada documento, el análisis de co-ocurrencia de palabras se basa en el contenido del propio documento. Utilizando como unidad de análisis todas las palabras clave, VOSviewer identificó un total de 2.414 términos, que se reducen a 2.026 una vez eliminadas las duplicidades aplicando el tesauro (por ejemplo: smart phone, smartphones, smartphone). La

Figura 2 representa un mapa de red con las 113 palabras clave que aparecieron conjuntamente 5 o más veces y después de deseleccionar 2 palabras (turismo y turista) por su carácter retórico y su escasa aportación al objeto de estudio.

Figura 2: Mapa de co-ocurrencia de palabras

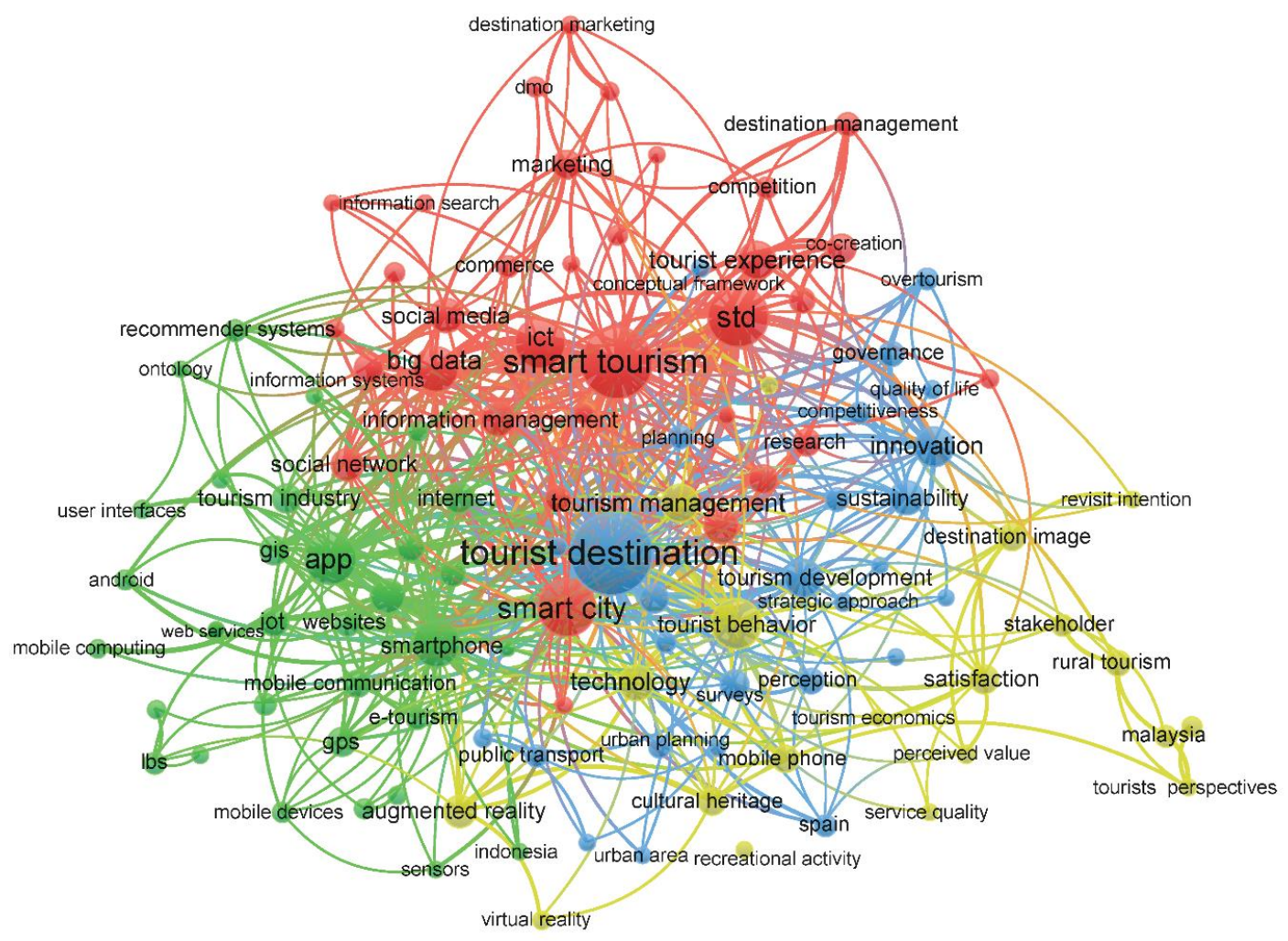

Elaboración propia 
Cada nodo representa una palabra clave y su tamaño proporciona una indicación relativa de la frecuencia de co-ocurrencia. Las palabras con mayor número de co-ocurrencias son tourist destination (118), smart tourism (87), smart tourism destination (std) (65), smart city (56), app (47), information and communication technologies (ict) (45), tourist behavior (43), smartphone (38), big data (36) e innovation (27). Palabras como tourist destination o smart tourism están situadas en el centro de la red, junto con otras palabras clave comunes, mientras que otras menos frecuentes están situadas en la periferia.

La red confirma una serie de fuertes vínculos entre determinadas palabras clave. El emparejamiento más fuerte es entre smart city y smart tourism, hecho que refuerza la idea de la importante relación entre estos dos conceptos. Los vínculos entre tourist behavior y smart city con tourist destination también son particularmente fuertes. Asimismo, existe una fuerte conexión entre smart tourism y smart city con smart tourism destination (std) y de information and communication technologies (ict) con smart tourism y smart tourism destination (std).

El mapa de co-ocurrencia de palabras sugiere 4 temas clave. El clúster 1 (rojo), contiene 34 palabras agrupadas en torno al concepto "smart". Se trata de un grupo con palabras clave que tienen una frecuencia de co-ocurrencia elevada como smart tourism, smart tourism destination (std), smart city, information and communication technologies (ict) y big data. Señalar la proximidad entre smart tourism destination (std) y tourist experience, evidenciando una de las claves de los destinos turísticos inteligentes: aumentar la calidad de la experiencia del visitante. El clúster 2 (verde), con un total de 32 palabras clave, pivota alrededor de las "tecnologías móviles". Incluye palabras como app, smartphone, internet, gis, gps e internet of things (iot). El tamaño y la coherencia de los nodos de este grupo, así como la densidad de enlaces a los otros grupos, dan una idea de su importancia dentro de la literatura sobre destinos turísticos inteligentes. El clúster 3 (azul) sugiere el tema de "gestión estratégica del destino". Contiene 27 palabras clave agrupadas en torno a tourist destination entre las que se encuentran tres de los cinco ejes sobre los que se apoyan los destinos turísticos inteligentes: innovation, sustanaibility y governance, además de otras relevantes como tourism development, planning y competitiveness. El clúster 4 (amarillo), es el grupo más pequeño con 20 palabras entre las que destaca tourist behavior. En él hay una gran variedad de palabras relacionadas con este concepto como satisfaction, technology y destination image. Los nodos de este grupo tienden a entremezclarse con los del clúster 3 (azul) e incluso los del 1 (rojo). Esta superposición parece indicar la estrecha relación del conocimiento del comportamiento turístico (antes, durante y después del viaje) con la comercialización del destino y su gestión estratégica.

Adicionalmente, el análisis temporal de la co-ocurrencia de palabras ofrece información sobre la evolución de los principales temas de interés (Figura 3). 
Figura 3: Análisis temporal de la co-ocurrencia de palabras

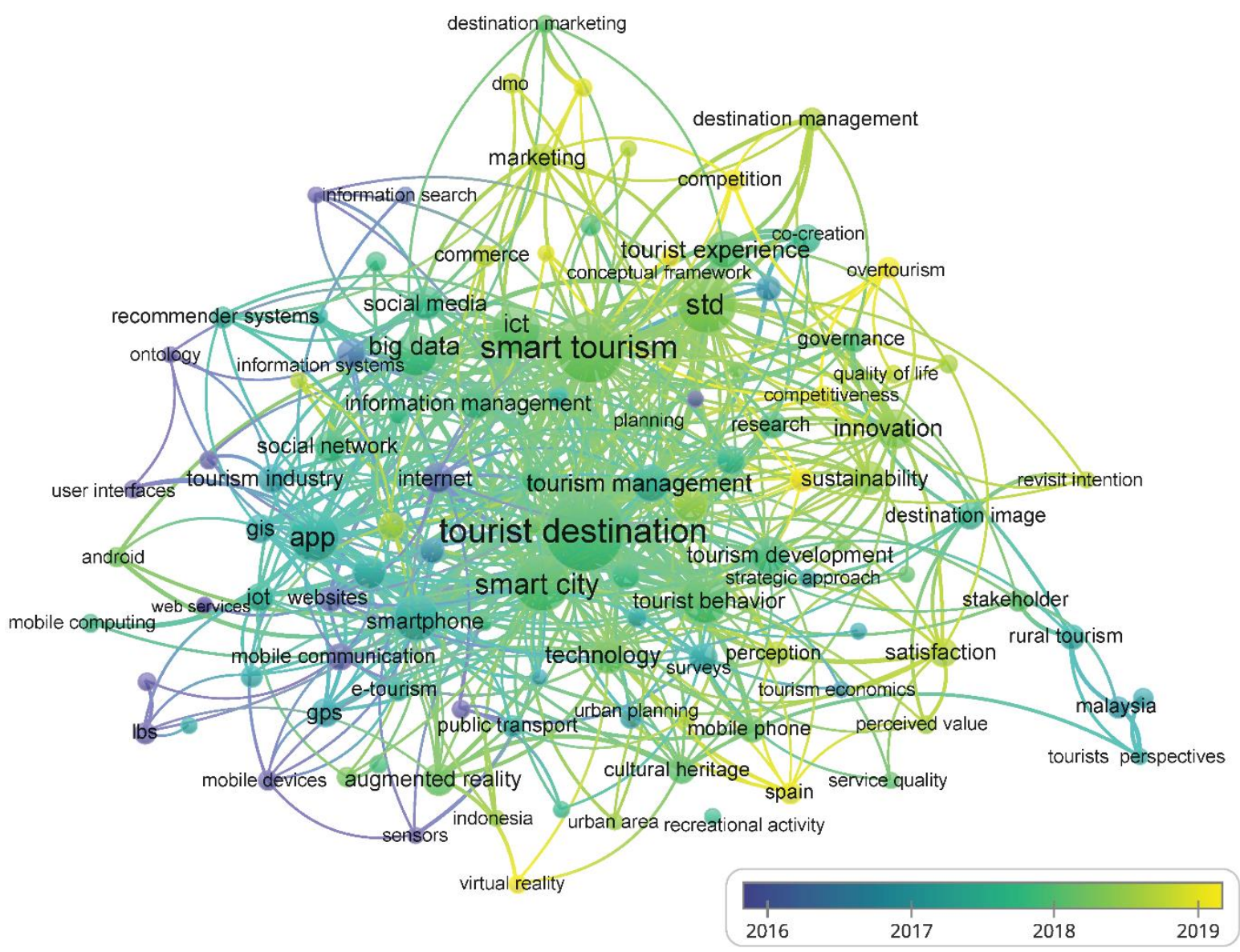

Elaboración propia

Los trabajos iniciales (nodos de color azul oscuro) tendían a centrarse en temas relacionados con la tecnología con términos como internet, information systems, mobile communication o websites. A partir de 2017 la tecnología sigue estando presente con palabras como app, smartphone, gis, internet of things (iot) y gps, pero aparecen otras más centrales y relacionadas con la gestión como tourism management. En 2018 aumenta considerablemente la frecuencia de co-ocurrencia, con palabras que constituyen el núcleo central de la red como tourist destination, smart tourism, smart tourism destination (std) y smart city. Las palabras clave de color verde más claro a amarillo se asocian con los últimos estudios y constituyen el "frente de investigación" (Price, 1965). Términos como satisfaction, marketing, perception, destination management, competitiveness, competition y overtourism son los más recientes.

\section{DISCUSIÓN}

Este trabajo emplea técnicas de análisis bibliométrico para analizar la producción científica asociada al turismo inteligente poniendo el foco en el destino, frente a investigaciones previas, de corte más generalista (Johnson y Samakovlis, 2019; Mehraliyev et al., 2020) o centradas en otros aspectos como las redes sociales (Vargas-Sánchez y Saltos, 2019) o los medios de comunicación sociales (Nusair, 2020). Además, está especialmente orientado hacia la visualización de los resultados mediante mapas bibliométricos. 
La investigación sobre destinos inteligentes ha tenido un rápido crecimiento (pregunta de investigación 1), sobre todo a partir de 2015 cuando surgen las definiciones más aceptadas. Este resultado es coherente con el obtenido en Bastidas-Manzano et al. (2020) o en Gretzel, Sigala, et al. (2015), para los que el turismo inteligente se convertido en una palabra de moda.

Este estudio también aporta información acerca de la distribución geográfica del conocimiento sobre destinos inteligentes (pregunta de investigación 2). Los resultados evidencian un importante desequilibrio, ya que la mayoría de los trabajos proceden de países desarrollados altamente digitalizados, sobre todo europeos y asiáticos. De cara al futuro sería interesante contar con más publicaciones de países en desarrollo que enriquezcan la literatura y proporcionen otros puntos de vista.

El análisis de citación ha identificado las publicaciones, autores y documentos más influyentes (pregunta de investigación 3). Journal of Destination Marketing and Management es el principal referente, aunque existen revistas donde se han publicado muy pocos trabajos pero que han tenido un gran impacto. Un número reducido de autores, entre los que sobresalen Chulmo Koo y Ulrike Gretzel, firman la mayoría de los trabajos. En cuanto a la "lista de lectura" recomendada, destaca el acreditado artículo de Gretzel, Sigala, et al. (2015).

La exploración de los patrones de co-citación de autores ha dado respuesta a la pregunta de investigación 4 acerca de la estructura intelectual de la literatura. Los autores se agrupan en 4 clústeres focalizados hacia las tecnologías de la información aplicadas al turismo, la gestión y la planificación estratégica, el destination marketing y otros más periféricos vinculados a la geografía y el territorio. Los destinos inteligentes parecen haber despertado un mayor interés en los autores que trabajan en tecnologías aplicadas al turismo y menos entre los que se ocupan de áreas tan relevantes para un destino como es la sociedad o el propio territorio en el que se asienta.

En relación con la pregunta 5 , el análisis de co-ocurrencia de palabras clave revela que la investigación está impulsada por 4 temas: el paradigma smart, las tecnologías móviles, la gestión estratégica del destino, y el comportamiento turístico. Los resultados respaldan la imbricación entre destinos inteligentes y la implementación de las tecnologías de la comunicación como eje central en su gestión, de acuerdo con Buhalis y Amaranggana (2015).

Del análisis de las palabras clave también se desprende que la accesibilidad, tanto física como digital (Invat.tur, 2015), es el eje que menos atención ha recibido de la academia, más centrada en la innovación o la tecnología. También hay una ausencia de investigaciones enfocadas hacia el desarrollo de herramientas o indicadores que proporcionen una medida del desempeño de los destinos a la hora de implementar una estrategia smart.

Los principales temas de interés han evolucionado desde aspectos tecnológicos muy generales hasta la literatura más reciente, que sitúa términos como satisfaction, marketing, perception, destination management, competitiveness, competition y overtourism como emergentes, revelando un interés creciente por la gestión, la mejora de la experiencia turística y la competitividad del destino e incluso por la sostenibilidad.

A pesar de todo, este tipo de análisis no está exento de limitaciones. Una de las principales es la elección de los términos de búsqueda en las bases de datos. Aunque se elijan cuidadosamente, pueden incluirse documentos que no están dentro del alcance del estudio, introduciendo valores atípicos y reduciendo la validez de los resultados (Zupic y Čater, 2015). 
Para filtrar los documentos no deseados se han establecido umbrales mínimos para los análisis de co-citación de autores y co-ocurrencia de palabras clave.

En este estudio se ha utilizado Scopus, una de las bases de datos con mayor cobertura en el ámbito del turismo (Hall, 2011; McKercher, 2007). Sin embargo, la eventual literatura no indexada en Scopus no está cubierta, existiendo la posibilidad de haber excluido publicaciones relevantes. En posteriores investigaciones sería interesante completar la búsqueda con registros de Web of Science.

El análisis de citación estima la influencia de las publicaciones, autores o documentos a través del número de citas que han producido. Su inconveniente es que las publicaciones más recientes han tenido menos tiempo de ser citadas, por lo que es una medida sesgada hacia las más antiguas. La co-citación es uno de los métodos bibliométricos más utilizados y validados (Miguel et al., 2007), pero presenta la misma desventaja.

La co-ocurrencia de palabras utiliza el contenido real de los documentos frente a otros métodos que sólo utilizan metadatos. La dificultad en este caso estriba en que las palabras pueden aparecer de diferentes maneras y tener distintos significados. Por ello, en este artículo se ha construido un tesauro para salvar este obstáculo y eliminar las posibles duplicidades.

\section{CONCLUSIONES}

El enfoque sistémico de los destinos turísticos inteligentes plantea una estrategia basada en la gobernanza, la sostenibilidad, la accesibilidad, la innovación y la tecnología como ejes vertebradores. Suponen un modelo de gestión que redunda en un incremento de la competitividad, un aumento de la eficiencia de los procesos de producción y comercialización, un estímulo al desarrollo sostenible, una mejora de la experiencia de los visitantes y de la calidad de vida de los residentes y, en definitiva, la dinamización económica del territorio.

A pesar de ser un concepto relativamente novedoso, ha tenido un rápido crecimiento en la investigación en turismo. El análisis bibliométrico es una buena herramienta para identificar y caracterizar la investigación sobre destinos turísticos inteligentes. Su principal fortaleza se basa en que es un proceso sistemático, directo y fácilmente reproducible, que minimiza el componente subjetivo del investigador e incrementa el rigor en las revisiones de la literatura.

Los mapas bibliométricos o mapas del conocimiento generados a partir de la cocitación de autores y de la co-ocurrencia de palabras facilitan el análisis de la complejidad de literatura sobre destinos turísticos inteligentes, de manera que permiten visualizar de forma gráfica las interrelaciones entre los diferentes elementos que lo forman.

Finalmente, es preciso reflexionar sobre el impacto de la crisis sanitaria generada por la COVID-19 en el turismo, ya que está siendo una de las actividades más afectadas por la pandemia. Estamos ante un escenario de gran incertidumbre, con un turista que tiene nuevas pautas de comportamiento que será preciso conocer y atender. En este sentido, los destinos turísticos inteligentes se configuran como un instrumento adecuado para mantener la competitividad de las empresas y hacer los destinos más seguros y sostenibles, gestionando los flujos de personas, controlando los aforos o evitando la saturación de espacios. Además, existe una gran variedad de recursos tecnológicos que pueden convertirse en un aliado para empresas y destinos, capaces a la vez de proporcionar a los turistas experiencias únicas y memorables personalizadas, desde las relacionadas con el internet de las cosas (IoT), big data, 
inteligencia artificial, realidad virtual o gamificación, hasta otras vinculadas con la gestión eficiente del agua, la calidad del aire, la energía o los residuos.

\section{REFERENCIAS BIBLIOGRÁFICAS}

Aguinis, H., Ramani, R. S., y Alabduljader, N. (2018). What you see is what you get? Enhancing methodological transparency in management research. Academy of Management Annals, 12(1), 83-110. https://doi.org/10.5465/annals.2016.0011

Albino, V., Berardi, U., y Dangelico, R. M. (2015). Smart cities: Definitions, dimensions, performance, and initiatives. Journal of Urban Technology, 22(1), 3-21. https://doi.org/10.1080/10630732.2014.942092

Bakici, T., Almirall, E., y Wareham, J. (2013). A Smart City Initiative: The Case of Barcelona. Journal of the Knowledge Economy, 4(2), 135-148. https://doi.org/10.1007/s13132$\underline{012-0084-9}$

Ballina, F. J., Valdés, L., y Del Valle, E. (2019). The Phygital experience in the smart tourism destination. International Journal of Tourism Cities, 5(4), 656-671. https://doi.org/10.1108/IJTC-11-2018-0088

Bastidas-Manzano, A. B., Sánchez-Fernández, J., y Casado-Aranda, L. A. (2020). The past, present, and future of Smart Tourism Destinations: A Bibliometric Analysis. Journal of Hospitality and Tourism Research, 45(3), 529-552. https://doi.org/10.1177/1096348020967062

Benckendorff, P. (2009). Themes and trends in Australian and New Zealand tourism research: A social network analysis of citations in two leading journals (1994-2007). Journal of Hospitality and Tourism Management, 16(1), 1-15. https://doi.org/10.1375/ihtm.16.1.1

Benckendorff, P., y Zehrer, A. (2013). A network analysis of tourism research. Annals of Tourism Research, 43, 121-149. https://doi.org/10.1016/i.annals.2013.04.005

Boes, K., Buhalis, D., y Inversini, A. (2015). Conceptualising Smart Tourism Destination Dimensions. En I. Tussyadiah y A. Inversini (Eds.), Information and Communication Technologies in Tourism 2015 (pp. 391-403). Springer International Publishing. https://doi.org/10.1007/978-3-319-14343-9 29

Boes, K., Buhalis, D., y Inversini, A. (2016). Smart tourism destinations: ecosystems for tourism destination competitiveness. International Journal of Tourism Cities, 2(2), 108-124. https://doi.org/10.1108/IJTC-12-2015-0032

Brandão Cavalheiro, M., Joia, L. A., y Do Canto Cavalheiro, G. M. (2019). Towards a Smart Tourism Destination Development Model: Promoting Environmental, Economic, Sociocultural and Political Values. Tourism Planning and Development, 1-23. https://doi.org/10.1080/21568316.2019.1597763

Buhalis, D., y Amaranggana, A. (2013). Smart Tourism Destinations. En Z. Xiang y I. Tussyadiah (Eds.), Information and Communication Technologies in Tourism 2014 (pp. 553-564). Springer International Publishing Switzerland. https://doi.org/10.1007/978-3-319$\underline{03973-2 \quad 40}$ 
Buhalis, D., y Amaranggana, A. (2015). Smart Tourism Destinations Enhancing Tourism Experience Through Personalisation of Services. En I. Tussyadiah y A. Inversini (Eds.), Information and Communication Technologies in Tourism 2015 (pp. 377-389). Springer International Publishing Switzerland. https://doi.org/10.1007/978-3-319-14343-9 28

Buhalis, D., y Foerste, M. (2015). SoCoMo marketing for travel and tourism: Empowering cocreation of value. Journal of Destination Marketing and Management, 4(3), 151-161. https://doi.org/10.1016/i.jdmm.2015.04.001

Buhalis, D., y Leung, R. (2018). Smart hospitality-Interconnectivity and interoperability towards an ecosystem. International Journal of Hospitality Management, 71, 41-50. https://doi.org/10.1016/j.ijhm.2017.11.011

Callon, M., Courtial, J.-P., Turner, W. A., y Bauin, S. (1983). From translations to problematic networks: An introduction to co-word analysis. Social Science Information, 22(2), 191235. https://doi.org/10.1177/053901883022002003

Caragliu, A., Del Bo, C., y Nijkamp, P. (2011). Smart cities in Europe. Journal of Urban Technology, 18(2), 65-82. https://doi.org/10.1080/10630732.2011.601117

Celdrán-Bernabeu, M. A., Mazón, J. N., Ivars-Baidal, J. A., y Vera-Rebollo, J. F. (2018). Smart Tourism. Un estudio de mapeo sistemático. Cuadernos de Turismo, 41, 107-138. https://doi.org/http://dx.doi.org/10.6018/turismo.41.326971

Chourabi, H., Nam, T., Walker, S., Gil-García, J. R., Mellouli, S., Nahon, K., Pardo, T. A., y Scholl, H. J. (2012). Understanding smart cities: An integrative framework. Proceedings of the 45th Hawaii International Conference on System Sciences, 2289-2297. https://doi.org/10.1109/HICSS.2012.615

Chung, N., Han, H., y Joun, Y. (2015). Tourists' intention to visit a destination: The role of augmented reality (AR) application for a heritage site. Computers in Human Behavior, 50, 588-599. https://doi.org/10.1016/i.chb.2015.02.068

Cobo, M. J., López-Herrera, A. G., Herrera-Viedma, E., y Herrera, F. (2012). SciMAT: A New Science Mapping Analysis Software Tool. Journal of the American Society for Information Science and Technology, 63(8), 1609-1630. https://doi.org/10.1002/asi.22688

Del Chiappa, G., y Baggio, R. (2015). Knowledge transfer in smart tourism destinations: Analyzing the effects of a network structure. Journal of Destination Marketing and Management, 4(3), 145-150. https://doi.org/10.1016/i.jdmm.2015.02.001

Dickinson, J. E., Ghali, K., Cherrett, T., Speed, C., Davies, N., y Norgate, S. (2014). Tourism and the smartphone app: capabilities, emerging practice and scope in the travel domain. Current Issues in Tourism, 17(1), 84-101. https://doi.org/10.1080/13683500.2012.718323

Exceltur. (2018). Estudio sobre el empleo en el sector turístico español. Exceltur. https://www.exceltur.org/wp-content/uploads/2018/04/ESTUDIO-EMPLEO-SECTORTURISTICO-EXCELTUR.pdf 
Giffinger, R., Fertner, C., Kramar, H., Kalasek, R., Meijers, E., y Pichler-Milanović, N. (2007). Smart cities: ranking of European mid-sized cities. Centre of Regional Science. http://www.smartcities.eu/download/smart cities final report.pdf

Gretzel, U., Sigala, M., Xiang, Z., y Koo, C. (2015). Smart tourism: foundations and developments. Electronic Markets, 25(3), 179-188. https://doi.org/10.1007/s12525$\underline{015-0196-8}$

Gretzel, U., Werthner, H., Koo, C., y Lamsfus, C. (2015). Conceptual foundations for understanding smart tourism ecosystems. Computers in Human Behavior, 50, 558-563. https://doi.org/10.1016/i.chb.2015.03.043

Hall, C. M. (2011). Publish and perish? Bibliometric analysis, journal ranking and the assessment of research quality in tourism. Tourism Management, 32(1), 16-27. https://doi.org/10.1016/i.tourman.2010.07.001

Instituto Nacional de Estadística. (2020). Notas de prensa. Cuenta Satélite del Turismo de España (CSTE). Revisión estadística 2019. Serie 2016-2019. https://www.ine.es/prensa/cst 2019.pdf

Invat.tur. (2015). Destinos Turísticos Inteligentes. Manual operativo para la configuración de Destinos Turísticos Inteligentes. Instituto Valenciano de Tecnologías Turísticas.

Ivars-Baidal, J. A., y Vera-Rebollo, J. F. (2019). Planificación turística en España. De los paradigmas tradicionales a los nuevos enfoques: planificación turística inteligente. Boletín de la Asociación de Geógrafos Españoles, 82, 1-31. https://doi.org/10.21138/bage.2765

Johnson, A.-G., y Samakovlis, I. (2019). A bibliometric analysis of knowledge development in smart tourism research. Journal of Hospitality and Tourism Technology, 10(4), 600-623. https://doi.org/10.1108/JHTT-07-2018-0065

Jovicic, D. Z. (2016). Key issues in the conceptualization of tourism destinations. Tourism Geographies, 18(4), 445-457. https://doi.org/10.1080/14616688.2016.1183144

Jovicic, D. Z. (2019). From the traditional understanding of tourism destination to the smart tourism destination. Current Issues in Tourism, 22(3), 276-282. https://doi.org/10.1080/13683500.2017.1313203

Koo, C., Shin, S., Gretzel, U., Hunter, W. C., y Chung, N. (2016). Conceptualization of Smart Tourism Destination Competitiveness. Asia Pacific Journal of Information Systems, 26(4), 367-384. https://doi.org/10.14329/apjis.2016.26.4.367

Koseoglu, M. A., Rahimi, R., Okumus, F., y Liu, J. (2016). Bibliometric studies in tourism. Annals of Tourism Research, 61, 180-198. https://doi.org/10.1016/i.annals.2016.10.006

Lamsfus, C., Martín, D., Alzua-Sorzabal, A., y Torres Manzanera, E. (2015). Smart tourism destinations: An extended conception of smart cities focusing on human mobility. En I. Tussyadiah y A. Inversini (Eds.), Information and Communication Technologies in Tourism 2015 (pp. 363-375). Springer International Publishing. https://doi.org/10.1007/978-3-319-14343-9 27

López de Ávila, A., y García, S. (2013). Destinos turísticos inteligentes. Economía Industrial, 395, 61-69. 
Lotka, A. J. (1926). The frequency distribution of scientific productivity. Journal of the Washington Academy of Sciences, 16(12), 317-323. http://www.jstor.org/stable/24529203

Mariné-Roig, E., y Antón Clavé, S. (2015). Tourism analytics with massive user-generated content: A case study of Barcelona. Journal of Destination Marketing and Management, 4(3), 162-172. https://doi.org/10.1016/i.jdmm.2015.06.004

McDonald, N. C. (2015). Are Millennials Really the "Go-Nowhere" Generation? Journal of the American Planning Association, 81(2), 90-103. https://doi.org/10.1080/01944363.2015.1057196

McKercher, B. (2007). A Study of Prolific Authors in 25 Tourism and Hospitality Journals. Journal of Hospitality \& Tourism Education, 19(2), 23-30. https://doi.org/10.1080/10963758.2007.10696887

Mehraliyev, F., Chan, I. C. C., Choi, Y., Koseoglu, M. A., y Law, R. (2020). A state-of-the-art review of smart tourism research. Journal of Travel \& Tourism Marketing, 37(1), 78-91. https://doi.org/10.1080/10548408.2020.1712309

Miguel, S., Caprile, L., y Jorquera-Vidal, I. (2008). Análisis de co-términos y de redes sociales para la generación de mapas temáticos. El profesional de la Informacion, 17(6), 637646. https://doi.org/10.3145/epi.2008.nov.06

Miguel, S., Moya-Anegón, F., y Herrero-Solana, V. (2007). El análisis de co-citas como método de investigación en Bibliotecología y Ciencia de la Información. Investigación Bibliotecológica, $21(43)$, 139-155. http://www.memoria.fahce.unlp.edu.ar/art revistas/pr.7252/pr.7252.pdf

Neuhofer, B., Buhalis, D., y Ladkin, A. (2012). Conceptualising technology enhanced destination experiences. Journal of Destination Marketing and Management, 1(1-2), 36-46. https://doi.org/10.1016/j.jdmm.2012.08.001

Neuhofer, B., Buhalis, D., y Ladkin, A. (2014). A Typology of Technology-Enhanced Tourism Experiences. International Journal of Tourism Research, 16(4), 340-450. https://doi.org/10.1002/itr.1958

Nusair, K. (2020). Developing a comprehensive life cycle framework for social media research in hospitality and tourism: A bibliometric method 2002-2018. International Journal of Contemporary Hospitality Management, 32(3), 1041-1066. https://doi.org/10.1108/IJCHM-09-2019-0777

Perianes-Rodríguez, A., Waltman, L., y van Eck, N. J. (2016). Constructing bibliometric networks: A comparison between full and fractional counting. Journal of Informetrics, 10(4), 1178-1195. https://doi.org/10.1016/i.joi.2016.10.006

Poslad, S., Laamanen, H., Malaka, R., Nick, A., Buckle, P., y Zipf, A. (2001). CRUMPET: Creation of user-friendly mobile services personalised for tourism. IEE Conference Publication, 477, 28-32. https://doi.org/10.1049/cp:20010006

Price, D. J. de S. (1965). Networks of Scientific Papers. Science, 149(3683), 510-515. https://doi.org/10.1126/science.149.3683.510 
Pritchard, A. (1969). Statistical bibliography or bibliometrics? Journal of Documentation, 25(4), 348-349.

Ramos-Rodríguez, A. R., y Ruiz-Navarro, J. (2004). Changes in the intellectual structure of strategic management research: A bibliometric study of the Strategic Management Journal, 1980-2000. Strategic Management Journal, 25(10), 981-1004. https://doi.org/10.1002/smj.397

Ruhanen, L., Weiler, B., Moyle, B. D., y McLennan, C. J. (2015). Trends and patterns in sustainable tourism research: a 25-year bibliometric analysis. Journal of Sustainable Tourism, 23(4), 517-535. https://doi.org/10.1080/09669582.2014.978790

Segittur. (2015). Informe destinos turísticos inteligentes: construyendo el futuro. Sociedad Estatal para la Gestión de la Innovación y las Tecnologías Turísticas. https://www.segittur.es/opencms/export/sites/segitur/.content/galerias/descargas/ proyectos/Libro-Blanco-Destinos-Tursticos-Inteligentes-ok_es.pdf

Shoval, N., y Birenboim, A. (2019). Customization and augmentation of experiences through mobile technologies: A paradigm shift in the analysis of destination competitiveness. Tourism Economics, 25(5), 661-669. https://doi.org/10.1177/1354816618806428

Spinak, E. (1996). Diccionario Enciclopédico de Bibliometría, Cienciometría e Informetría. UNESCO.

Van Eck, N. J., y Waltman, L. (2010). Software survey: VOSviewer, a computer program for bibliometric mapping. Scientometrics, 84(2), 523-538. https://doi.org/10.1007/s11192-009-0146-3

Van Eck, N. J., y Waltman, L. (2014). Visualizing Bibliometric Networks. En Y. Ding, R. Rousseau, y D. Wolfram (Eds.), Measuring scholarly impact: Methods and practice (pp. 285-320). Springer International Publishing Switzerland. https://doi.org/10.1007/978-3-31910377-8 13

Vargas-Sánchez, A., y Saltos, A. E. (2019). Smartness and social networks as shapers of the tourism industry: What is being done in Academia in this intersection? Worldwide Hospitality and Tourism Themes, 11(6), 748-759. https://doi.org/10.1108/WHATT-09$\underline{2019-0057}$

Wang, D., Li, X. R., y Li, Y. (2013). China's «smart tourism destination» initiative: A taste of the service-dominant logic. Journal of Destination Marketing and Management, 2(2), 5961. https://doi.org/10.1016/i.jdmm.2013.05.004

White, H. D., y Griffith, B. C. (1981). Author cocitation: A literature measure of intellectual structure. Journal of the American Society for Information Science, 32(3), 163-171. https://doi.org/10.1002/asi.4630320302

World Tourism Organization. (2021). International Tourism Highlights, 2020 Edition. UNWTO. https://doi.org/10.18111/9789284422456

Xiang, Z., Tussyadiah, I., y Buhalis, D. (2015). Smart destinations: Foundations, analytics, and applications. Journal of Destination Marketing and Management, 4(3), 143-144. https://doi.org/10.1016/j.jdmm.2015.07.001 
Zupic, I., y Čater, T. (2015). Bibliometric Methods in Management and Organization. Organizational Research Methods, 18(3), 429-472. https://doi.org/10.1177/1094428114562629

\section{CONTRIBUCIONES DE LOS AUTORES:}

Autor 1: concepción original del trabajo; análisis, adquisición e interpretación de los datos; redacción y revisión crítica del contenido y; aprobación final de la versión a publicar.

Autor 2: redacción y revisión crítica del contenido y; aprobación final de la versión a publicar. Autor 3: redacción y revisión crítica del contenido y; aprobación final de la versión a publicar. 